\title{
Finanzdienstleistung im Verbund
}

\author{
Wilfried Theißen-Boljahn
}

Gute soziale Ideen sollen nicht am fehlenden Startkapital scheitern. Die Auffassung führte vor zwei Jahrzehnten zur Gründung der Paritätischen Geldberatung eG in Nordrhein-Westfalen.

Schon vor zwanzig Jahren war das Geld für soziale Projekte knapp. Und dies vor allem dann, wenn neue Ideen und bürgerschaftliches Engagement auf zwar verständnisvolles, aber dennoch skeptisches Achselzucken bei den klassischen Banken und Kreditinstituten stieß. Mit der Gründung einer Geldberatungs-Genossenschaft hat der Paritätische Wohlfahrtsverband in Nordrhein-Westfalen bereits 1986 ein neues Finanzierungsinstrument für seine mehr als 3.000 Mitgliedsorganisationen geschaffen, um innovative Projekte auf den Weg zu bringen.

»Die Grundidee war, den Menschen die Möglichkeit zu geben, sich mit ihrem Sparbuch und mit Genossenschaftsanteilen aktiv in die Sozialpolitik einzumischen und mit ihren Einlagen ihr Geld sozial arbeiten zu lassen «, erinnert sich der Aufsichtsratsvorsitzende Dr. Jörg Steinhausen, Landesgeschäftsführer des Paritätischen in Nordrhein-Westfalen.

\section{Kooperation zwischen Bank und Wohlfahrtsverband}

Immer knappere öffentliche Mittel unterstreichen die Wichtigkeit der Idee. Sowohl die Veränderungen, die sich durch fehlende Mittel ergeben, aber auch Veränderung am Kapitalmarkt, beispielsweise durch »Basel II«, erfordern inzwischen intelligente und flexible Finanzierungskonzepte.

Die Kooperation mit der Bank für Sozialwirtschaft ermöglichte die Einrichtung des Spar- und Kreditfonds, der wiederum zinsgünstige Darlehen ermöglicht. Über diese Darlehen hinaus arbeitet die Paritätische Geldberatung eG eng mit der Bank für Sozialwirtschaft zusammen, wenn es um Konsortialfinanzierungen oder auch die Bewältigung von akuten finanziellen Krisen geht.

Die paritätischen Sparbücher, deren Zinserträge ganz oder teilweise für soziale Projekte gespendet werden, bieten jedermann eine ethische Anlage auch mit kleinen Beträgen. Auf der örtlichen Ebene fungieren lokale »Fördervereine für Soziale Arbeit « als lokale Agentur für den Sparund Kreditfonds, und stärken durch die Zeichnung von Genossenschaftsanteilen die Paritätische Geldberatung. Über die Verzahnung von örtlicher Präsenz, überörtlichem Know-how und der Einbindung von Verband und Bank, ergibt sich eine passgenaue Finanzdienstleistung, die nie Gefahr läuft an den tatsächlichen Bedürfnissen vorbei zu gehen.

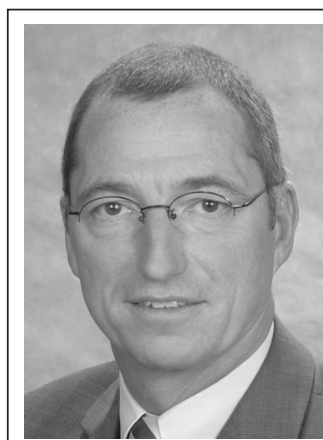

schulen.
Wilfried Theißen-Boljahn (51) ist seit 1992 Vorstand der Paritätischen Geldberatung eG und seit 1999 Vorstand der Stiftung Gemeinsam Handeln. Er hat Sozialpädagogik und Betriebswirtschaft für die Sozialwirtschaft studiert. Er hat Lehraufträge an verschiedenen Fachhoch-
Das Leistungsspektrum der Paritätischen Geldberatung eG umfasst die gesamte Beratung »Rund ums Geld«. Dazu gehören die Entwicklung von Finanzierungen, Darlehen und auch Zuschüsse. Seit der Gründung wurden fast 9,4 Millionen Euro Darlehen, aus dem Spar- und Kreditfonds und dem Genossenschaftskapital vergeben. Der Spar- und Kreditfonds hat aktuell ein Volumen von rund 3,2 Millionen Euro.

Soziales Engagement bedeutet dort zuzuhören, wo andere den Kopf schütteln. Es gilt gemeinsam kreative und innovative, aber abgesicherte Lösungen zu finden. Um ein Darlehen zu bekommen, können die Mitgliedsorganisationen des Paritätischen direkt bei den Mitarbeiterinnen und Mitarbeiter der Paritätischen Geldberatung oder bei jeder Geschäftsstelle des Paritätischen Wohlfahrtsverbandes in Nordrhein-Westfalen anrufen. Durch die enge Zusammenarbeit von Paritätischer Geldberatung, Verband und Bank für Sozialwirtschaft können so gemeinsam mit den Trägern regionale Lösungen gesucht werden, die durch die Nähe zum Projekt eine deutliche höhere Tragfähigkeit haben.

Auch bei den »paritätischen Darlehen « sind natürlich Sicherheiten gefordert. Das bekannte Problem bei vielen gemeinnützigen Vereinen ist jedoch das Fehlen »banküblicher « Sicherheiten. Schon seit vielen Jahren werden daher bei Darlehensvergaben entweder Sicherheitengemeinschaften oder Tilgungsgemeinschaften gebildet. Hierbei wird das Umfeld der Träger einbezogen. Über diesen Weg werden dann im Rahmen von privaten Bürgschaften die benötigten Darlehen abgesichert. So verbinden sich die Ideen von zeitgemäßem »Relationship-Fundrasing « und konventioneller Finanzierung miteinander. 


\section{Die Paritätische Geldberatung eG}

in Nordrhein-Westfalen wurde 1986 gegründet und ist eine Tochter des Paritätischen Wohlfahrtsverbandes, Landesverband Nordrhein-Westfalen e. V. Die Paritätische Geldberatung unterstützt soziale Initiativen und Träger bei Finanzierung Sozialer Arbeit und sozialer Projekte. Das Leistungsspektrum ist die umfassende Finanzberatung; dazu gehören alle Formen des Sparens, die Entwicklung von Finanzierungen, Darlehen und Zuschüssen. Ebenso gehört zu dem täglichen Aufgabenbereich die Beratung der etwa 3.000 dem Paritätischen angeschlossenen Mitgliedsorganisationen in Nordrhein-Westfalen. Paritätische Geldberatung eG, Loher Straße 7, 42283 Wuppertal, Telefon 0202 2822-342, Fax 0202 2822-344, E-Mail geldberatung@paritaet-nrw.org, Internet http://www.paritaetnrw.org

\section{Garantiefonds auf Gegenseitigkeit "La Garantie Solidaire«}

Der Garantiefonds auf Gegenseitigkeit »La Garantie Solidaire« wurde auf europäischer Ebene eingerichtet. Getragen wird er von Finanzinstituten, die der Europäischen Förderung ethisch alternativer Banken angehören (FEBEA), in der auch die Bank für Sozialwirtschaft mitwirkt. Die Paritätische Geldberatung eG hat im vergangenen Jahr gemeinsam mit der Bank für Sozialwirtschaft eine Vereinbarung mit dem Garantiefonds »La Garantie Solidaire « getroffen, nach der die Paritätische Geldberatung nun in der Lage ist, ausgereichte Kredite bis zu 50 Prozent über diesen Sicherungsfonds abzusichern. Insgesamt sind im Fonds 50.000 Euro, mit dieser Summe können Mikrokredite bis zu 500.000 Euro abgesichert werden. Wir nutzen diese Form der Absicherung ausschließlich für Kredite bis 25.000 Euro und setzen dieses Instrument nur dann ein, wenn dem Projekt keine anderen banküblichen Sicherheiten zur Verfügung stehen.

Der Garantiefonds hilft bei fehlenden Sicherheiten insbesondere bei kleinen Organisationen, da er geringe zusätzliche Kosten verursacht. Die Möglichkeit gerade kleinere Volumen so abzusichern hat in der Praxis geholfen. So wurden einige ambulante Pflegedienste beim Ausbau ihres Leistungsspektrums und der Sicherung von Arbeitsplätzen gefördert.

\section{Finanzierungsmix statt Abhängigkeit von einer Finanzquelle}

Soziale Projekte sind heute immer mehr denn je auf einen intelligenten Finanzierungsmix angewiesen. Die Kombination von Mitteln der Kommunen und des Landes, Stiftungsmitteln, Kapitalmarktdarlehen und Instrumenten des Fundraising, charakterisieren heute die Finanzierung von sozialen Projekten. Die Paritätische Geldberatung verbindet daher bei der Entwicklung von Finanzierungskonzepten immer verschiedene Quellen und unterstützt die Träger bei der Entwicklung von eigenen Fundraising-Konzepten.

»Ohne Moos nichts los.« Ein alter Spruch, der aber immer noch aktuell ist. Schon in den neunziger Jahren wurde das Fundraising populär. Wer hat für seine Institution nicht schon mal Spenden bekommen. Ob Bußgelder oder Stiftungsgelder alles bekannte Geldquellen. Vom
Flohmarkt bis zum Benefizkonzert: keine Geheimnisse. Auch Sozialmarketing und Sozialsponsoring sind keine ganz neuen Begriffe in sozialen Einrichtungen.

Aber Fundraising ist eben mehr als nur die isolierte Anwendung einzelner Instrumente. Erfolg hat mittel- und langfristig nur diejenige Institution, der es gelingt, Fundraising als festen Bestandteil in die Gesamtorganisation einzubinden. Das heißt, Fundraising muss in Zukunft integraler Bestandteil der Öffentlichkeitsarbeit und der Organisations- und Projektentwicklung werden. Das eigentliche Fundraising beginnt erst dann, wenn auch daran gedacht wird den Förderern und Unterstützern zu danken und sie mittelund langfristig an die Einrichtung zu binden.

\section{Ein Musterbeispiel für aktive Geldberatung: Die Obdachlosenzeitung »fiftyfifty»}

Als Ende 1994 der Verein Asphalt e. V. in Düsseldorf ein Straßenmagazin initiieren wollte, waren viele skeptisch. Eine Zeitung von Obdachlosen gemacht? Eine Zeitung, die von Obdachlosen auf der Straße verkauft wird? Eine Zeitung, die eine Alternative zum Betteln sein soll? Aus den Mitteln der Paritätischen Geldberatung wurde dann im Februar 1995 ein Zuschuss bewilligt. Mit diesen 2.556,46 Euro (5.000 DM) und dem Engagement der Aktiven bei Asphalt e. V. wurde »fiftyfifty« an den Start gebracht.

Zehn Jahre später ist aus dieser Idee und dem kleinen Zuschuss eine viel beachtete und für die Betroffenen effektive Organisation geworden. Das Straßenmagazin »fiftyfifty « ist mittlerweile eine feste Größe und bietet vielen eine menschenwürdige Alternative zum Betteln. Durch »fiftyfifty« konnten fast 2.000 Obdachlose in neuen Wohnungen untergebracht werden. Inzwischen gibt es Projekte der Armenspeisung, Trainingswohnungen für obdachlose Frauen, eine Notschlafstelle für minderjährige, drogensüchtige Prostituierte und vieles mehr.

Die »fiftyfifty«-Galerie macht weit über die Grenzen Düsseldorfs von sich reden. Die Kunst dient hier in erster Linie dem Wohle der Obdachlosen. Die Liste der Künstler, die »fiftyfifty « unterstützen, liest sich wie das »Who is who" der deutschen Gegenwartskunst. Von Jörg Immendorf bis Günther Uecker, von Thomas Ruff bis Fritz Schwelger, alle Künstler stellen Werke zur Verfügung. Die Galerie ist inzwischen ein Geheimtipp für Schnäppchenjäger und über das Internet wird bis in die USA mit Kunst gehandelt.

Viel hilft viel — in diesem Fall ist aus einem kleinen $\mathrm{Zu}$ schuss ein Projekt geworden, das vielen viel hilft.

\section{Resümee}

In den zurückliegenden Jahren hat sich insbesondere die enge Verzahnung der beteiligten Organisationen bewährt. Durch die immer enger werdende Situation der öffentlichen Kassen wird besonders die Entwicklung neuer und innovativer Projekte gebremst. Hier ist für die $\mathrm{Zu}$ kunft die größte Herausforderung zu sehen. Gleichzeitig gilt es vorhandene Strukturen zu sichern und zukunftssicher zu machen. 